\title{
Spinal versus general anesthesia for Cesarean section in patients with sickle cell anemia
}

\author{
Mohamed H. Bakri, Eman A. Ismail, Gamal Ghanem, and Mahmoud Shokry \\ Department of Anesthesia, Assiut University Faculty of Medicine, Assiut, Egypt
}

Background: Sickle cell anemia (SCA) increases the rate of maternal and fetal complications. This pilot study was designed to compare the maternal and fetal outcomes of spinal versus general anesthesia (GA) for parturients with SCA undergoing cesarean delivery.

Methods: Forty parturients with known SCA scheduled for elective Cesarean delivery were randomized into spinal anesthesia $(n=20)$ and GA groups $(n=20)$. Perioperative hemodynamic parameters were recorded. Postpartum complications were followed up. Opioid consumption was calculated. Blood loss during surgery and the number of patients who received intraoperative or postpartum blood transfusion were recorded. Patient satisfaction with the type of anesthesia was assessed. The Apgar score at 1 and $5 \mathrm{~min}$, neonatal admission to the intensive care unit, and mortality were also recorded. Results: Blood loss was significantly higher in the GA than spinal group $(P=0.01)$. However, the number of patients who received an intraoperative or postpartum blood transfusion was statistically insignificant. Significantly more patients developed intraoperative hypotension and bradycardia in the spinal than GA group. Opioid use during the first $24 \mathrm{~h}$ was significantly higher in the GA than spinal group $(\mathrm{P}<0.0001)$. More patients had vaso-occlusive crisis in the GA than spinal group without statistical significance $(\mathrm{P}=0.4)$. There was one case of acute chest syndrome in the GA group. No significant differences were observed in postoperative nausea and/or vomiting, patient satisfaction, or hospital length of stay. Neonatal Apgar scores were significantly better in the spinal than GA group at 1 and $5 \min (P=0.006$ and $P=0.009$, respectively). Neonatal intensive care admission was not significantly different between the two groups, and there was no neonatal mortality.

Conclusions: Spinal anesthesia may have advantages over GA in parturients with SCA undergoing Cesarean delivery.

Key Words: Apgar score, Cesarean section, General anesthesia, Sickle cell anemia, Spinal anesthesia.

Corresponding author: Eman A. Ismail, M.D.

Department of Anesthesia, Faculty of Medicine, Assiut University, 71515 Assiut, Egypt

Tel: 966504943551 , Fax: 966135755150

E-mail: emanismail97@gmail.com

Received: April 9, 2015.

Revised: May 6, 2015.

Accepted: May 11, 2015.

Korean J Anesthesiol 2015 October 68(5): 469-475

http://dx.doi.org/10.4097/kjae.2015.68.5.469

\section{Introduction}

Sickle cell disease refers to a group of disorders that produce abnormal hemoglobin S molecules. Sickle cell anemia (SCA) is the specific form of sickle cell disease characterized by homozygosity for hemoglobin S (HbSS genotype). During stressful situations, such as pregnancy and surgery, deoxygenation of hemoglobin molecules and sickling of many red blood cells may occur. This may cause vascular occlusion and tissue ischemia, resulting in painful crises that characterize SCA [1].

(c) This is an open-access article distributed under the terms of the Creative Commons Attribution Non-Commercial License (http://creativecommons.org/ licenses/by-nc/4.0/), which permits unrestricted non-commercial use, distribution, and reproduction in any medium, provided the original work is properly cited. 
Pregnancy in women with SCA is a high-risk situation, and the physiological changes that occur during pregnancy can overburden different organs that already have chronic injuries secondary to sickle cell disease with a subsequent increase in the rate of maternal and fetal complications [2]. Proper planning and optimal perioperative preparation are keys to the successful management of patients with sickle cell disease. There is no consensus regarding the choice of anesthetic technique for parturients with SCA because the impact of the anesthetic technique may affect the occurrence of sickling complications [3].

Controversy exists regarding the choice of spinal or general anesthesia (GA) for the management of patients with SCA undergoing Cesarean section (CS). There has been an increase in the number of pregnant patients with SCA; unfortunately, however, no prospective randomized studies, to the best of our knowledge, have compared the outcomes of spinal versus GA in parturients with SCA. Thus, this pilot study was designed to compare the maternal and fetal outcomes of spinal versus GA for parturients with SCA undergoing CS.

\section{Materials and Methods}

After obtaining local ethics committee approval and informed written consent from each patient, 40 pregnant women with known SCA (HbSS genotype) scheduled for elective CS were included in this study over a 32-month period. Patients were excluded if they had medical complications other than SCA, such as pregnancy-induced hypertension, antepartum hemorrhage, fetal abnormality, multiple pregnancies, or coagulopathies.

Patients were randomly allocated into two equal groups (20 patients each) according to computer-generated randomnumber sequences. The spinal group $(n=20)$ underwent spinal anesthesia, and the GA group underwent GA $(n=20)$. Preoperative evaluation in both study groups included a detailed history, physical examination, and review of antenatal care, including laboratory investigations such as the complete blood cell count, renal and liver function tests, and coagulation profiling. All women were given intravenous (I.V.) ranitidine $(50 \mathrm{mg}$ ) and metoclopramide (10 $\mathrm{mg}$ ) before the induction of anesthesia and cefazolin sodium ( $1 \mathrm{~g})$ as a prophylactic antibiotic after umbilical cord clamping. Women in both groups were placed in the left $15^{\circ}$ lateral tilt position until delivery to protect against supine hypotension syndrome. After preoperative assessment and randomization, all of the patients received standard continuous monitoring of their electrocardiograph in leads II and V5, heart rate, arterial oxygen saturation measured by pulse oximetry, noninvasive blood pressure, end-tidal $\mathrm{CO}_{2}$ (for the GA group), and nasopharyngeal temperature. A forced-air warming blanket was used to maintain the temperature at $>36.0^{\circ} \mathrm{C}$ (Bair Hugger;
Arizant Medical, Eden Prairie, MN, USA).

All patients in the spinal group received an I.V. infusion of 15 $\mathrm{ml} / \mathrm{kg}$ lactated Ringer's solution before spinal anesthesia. With the patient in the sitting position and after identification of the L4-5 intervertebral space, the local anesthetic was infiltrated using $2 \mathrm{ml}$ of $1 \%$ plain lidocaine. Lumbar puncture was performed using the midline approach with a $25-\mathrm{G}$ Whitacre needle (pencil-point). A dose of 8 to $12 \mathrm{mg}$ of $0.5 \%$ heavy bupivacaine with $10 \mu \mathrm{g}$ of fentanyl was injected intrathecally. Next, patients were immediately placed in the recumbent position with left uterine displacement and a $15^{\circ}$ Trendelenburg position to optimize cephalic spread of the anesthetic drugs. Adequate anesthesia was defined as an upper sensory spread (absence of sensation to cold) to a level of T4. Hypotension (systolic blood pressure < $90 \mathrm{mmHg}$ ) was treated with boluses of fluids and/or ephedrine hydrochloride at 5 to $10 \mathrm{mg}$.

In the GA group, anesthetic management was performed using a standardized protocol. Patients received an I.V. infusion of $10 \mathrm{ml} / \mathrm{kg}$ lactated Ringer's solution before induction of anesthesia in the holding area. Next, after preoxygenation, anesthesia was induced with propofol at 2.0 to $2.5 \mathrm{mg} / \mathrm{kg}$ and atracurium besylate at $0.5 \mathrm{mg} / \mathrm{kg}$ to facilitate endotracheal intubation. After intubation, the patients were maintained on controlled mechanical ventilation adjusted to maintain an end-tidal carbon dioxide pressure of $35 \mathrm{mmHg}$. Anesthesia was maintained with a 1.0 to $2.0 \%$ end-tidal concentration of isoflurane with $100 \%$ oxygen. After delivery of the fetus, fentanyl was administered at $1 \mu \mathrm{g} /$ $\mathrm{kg}$. Subsequent incremental boluses of fentanyl at $0.5 \mu \mathrm{g} / \mathrm{kg}$ and atracurium besylate at $0.1 \mathrm{mg} / \mathrm{kg}$ were administered as clinically indicated. After surgical closure, I.V. atropine and neostigmine (1.0 and $2.5 \mathrm{mg}$, respectively) were administered slowly to restart spontaneous breathing, which was followed by tracheal extubation. Just after delivery of the fetus, an oxytocin infusion at a dose of $10 \mathrm{IU} / \mathrm{h}$ was administered to both groups. In the recovery area and thereafter, I.V. morphine was administered at a dose of $0.1 \mathrm{mg} / \mathrm{kg}$ for postoperative analgesia to all patients from both groups according to the visual analog scale of pain. The dose was given and repeated to maintain a visual analog scale score of $\leq 3$. The total amount of morphine during the first $24 \mathrm{~h}$ was calculated for both study groups. During the postpartum period, the patients were adequately hydrated, given adequate analgesia, and kept warm. Additionally, $40 \mathrm{mg}$ of prophylactic subcutaneous low-molecular-weight heparin (enoxaparin) was administered daily, and incentive spirometry was encouraged.

The mean blood loss volume during surgery and the number of patients who received an intraoperative or postpartum blood transfusion were recorded. For the purpose of the study, the mean arterial blood pressure and heart rate were recorded preoperatively and 5, 10, and $15 \mathrm{~min}$ after induction of anesthesia (or after administration of spinal anesthesia), followed by every 
15 min until 120 min from the start of anesthesia, and then every 30 min until $210 \mathrm{~min}$ from the start of anesthesia. All of the patients were followed up for any postpartum complications until they were discharged from the hospital. These complications included vaso-occlusive crises (obstruction of the microcirculation by sickled red blood cells, causing ischemia), acute chest syndrome (vaso-occlusive crisis affecting the lungs that most closely resembles pneumonia), postoperative nausea and vomiting (PONV), postdural puncture headache, and any other complications [4].

Immediately after delivery, all newborns were managed by neonatologists in another room connected to the operating room. The neonatologists who assessed the Apgar scores were blinded to the type of anesthetic technique used. The Apgar scores at 1 and $5 \mathrm{~min}$ were recorded for all of the neonates. The numbers of neonates who were admitted to the neonatal intensive care unit and who died after delivery until the mother was discharged from the hospital were also recorded.

Patient satisfaction with the type of anesthetic technique used was assessed at $24 \mathrm{~h}$ postoperatively using a verbal global satisfaction score comprising the five following categories: (1) very dissatisfied, (2) slightly dissatisfied, (3) neither satisfied nor dissatisfied, (4) satisfied, and (5) highly satisfied. For the purpose of the study, categories 1,2 , and 3 were considered "unsatisfied" and 4 and 5 "satisfied" [5].

\section{Statistical analysis}

Statistical analysis was performed using the Statistical Package for Social Sciences (SPSS) version 17.0 (SPSS Inc., Chicago, IL, USA). Descriptive statistics were derived for the study population and are expressed as the number, percentage, or mean \pm standard deviation as indicated. Data were analyzed and compared using Fisher's exact or the chi-squared $\left(\chi^{2}\right)$ test for categorical variables and the unpaired t test for continuous data. A P value of $<0.05$ was considered to indicate statistical significance.

\section{Results}

Table 1 shows the baseline clinical characteristics for both study groups. There were no statistically significant differences between the two groups in maternal age, gestational age, maternal weight, neonatal weight, operative duration, preoperative hemoglobin level, or reason for CS. The mean blood loss volume was significantly higher in the GA than spinal group $(P=0.01)$ (Table 2). However, the number of patients who received an intraoperative blood transfusion or postpartum transfusion was statistically insignificant. More patients developed intraoperative hypotension in the spinal than GA group $(\mathrm{P}=0.4)$ (Table 2 ). Three patients in the spinal group had bradycardia (heart rate $<60$ beats/min) that was associated with hypotension and improved by ephedrine. None of the patients in either group received intraoperative atropine, but the mean total use of intraoperative ephedrine was significantly higher in the spinal than GA group $(\mathrm{P}<0.0001)$ (Table 2$)$. Fig. 1 shows that the mean arterial blood pressure was significantly lower in the spinal than GA group during the first $15 \mathrm{~min}$ after anesthesia, but significantly higher in the spinal than GA group 30 and 45 min after anesthesia. On the other hand, the mean heart rate was significantly lower in the spinal than GA group only during the first $30 \mathrm{~min}$ after anesthesia (Fig. 2). However, the changes in blood pressure and heart rate were not clinically significant. None of the patients in the spinal group received I.V. fentanyl intraoperatively.

Table 1. Baseline Clinical Characteristics of Both Study Groups

\begin{tabular}{|c|c|c|}
\hline Variable & Spinal group $(n=20)$ & General anesthesia group $(\mathrm{n}=20)$ \\
\hline Maternal age (yr) & $27 \pm 4$ & $29 \pm 5$ \\
\hline Gestational age (weeks) & $37 \pm 1$ & $37 \pm 2$ \\
\hline Maternal weight $(\mathrm{kg})$ & $65 \pm 8$ & $63 \pm 5$ \\
\hline Neonatal weight $(\mathrm{g})$ & $2,625 \pm 350$ & $2,550 \pm 275$ \\
\hline Preoperative Hb level (g/dl) & $8.9 \pm 0.4$ & $9.1 \pm 0.6$ \\
\hline Operation duration (min) & $54 \pm 13$ & $51 \pm 16$ \\
\hline \multicolumn{3}{|l|}{ Causes of Cesarean section $(n)$ : } \\
\hline 1- Cephalopelvic disproportion & 9 & 7 \\
\hline 2- Breech presentation & 2 & 3 \\
\hline 3- Transverse lie & 2 & 2 \\
\hline 4- Placenta previa & 3 & 4 \\
\hline 5- Premature rupture of membranes with a previous Cesarean section & 3 & 2 \\
\hline 6- Two or more previous Cesarean sections & 1 & 2 \\
\hline
\end{tabular}

Values are mean \pm SD or number of patients. No significant differences $(\mathrm{P}>0.05)$ between the two groups in maternal age, gestational age, maternal weight, neonatal weight, operative duration, preoperative hemoglobin level, or the cause of Cesarean section. 
Table 2. Intraoperative and Postoperative Maternal Outcome Variables for Both Study Groups

\begin{tabular}{|c|c|c|c|}
\hline Variable & $\begin{array}{l}\text { Spinal group } \\
(\mathrm{n}=20)\end{array}$ & $\begin{array}{l}\text { General anesthesia group } \\
\qquad(\mathrm{n}=20)\end{array}$ & P value \\
\hline Mean blood loss during surgery $(\mathrm{ml})$ & $450 \pm 100$ & $525 \pm 75$ & 0.01 \\
\hline Number of patients who received intraoperative blood transfusion (n) & 3 & 5 & 0.6 \\
\hline Number of patients with hypotension during anesthesia (n) & 5 & 2 & 0.4 \\
\hline Mean total intraoperative ephedrine use (mg) & $5.5 \pm 2.5$ & 0 & $<0.0001$ \\
\hline Number of patients with bradycardia during anesthesia (n) & 3 & 0 & 0.2 \\
\hline Intraoperative intravenous use of fentanyl $(\mu \mathrm{g})$ & 0 & $62.5 \pm 10.6$ & $<0.0001$ \\
\hline \multicolumn{4}{|l|}{ Postoperative complications: $(n)$} \\
\hline 1- Postpartum transfusion & 1 & 3 & 0.6 \\
\hline 2- Vaso-occlusive crisis & 4 & 7 & 0.4 \\
\hline 2- Acute chest syndrome & 0 & 1 & 1.0 \\
\hline 4- Postoperative nausea and vomiting during the first $24 \mathrm{~h}$ postoperatively: & 3 & 7 & 0.3 \\
\hline a- vomiting & 1 & 3 & 0.6 \\
\hline b- nausea & 2 & 4 & 0.6 \\
\hline 5- Postdural puncture headache & 0 & 0 & 1.0 \\
\hline Mean total morphine requirement in the first $24 \mathrm{~h}$ postoperatively $(\mathrm{mg})$ & $7.4 \pm 2.6$ & $11.5 \pm 2.3$ & $<0.0001$ \\
\hline Patient satisfaction: & & & 0.5 \\
\hline a- Satisfied [n (\%)] & $15(75 \%)$ & $12(60 \%)$ & \\
\hline b- Unsatisfied [n (\%)] & $5(25 \%)$ & $8(40 \%)$ & \\
\hline Hospital length of stay after delivery (days) & $6.3 \pm 1.2$ & $7.2 \pm 1.8$ & 0.07 \\
\hline
\end{tabular}

Values are mean \pm SD or number of patients. No significant differences $(P>0.05)$ between study groups in the number of patients who received intraoperative or postpartum blood transfusion, or postoperative complications. There were significant differences $(\mathrm{P}<0.05)$ between study groups in the mean amount of blood loss during surgery, total amount of intra-operative fentanyl and ephedrine use, and postoperative morphine requirement in the first $24 \mathrm{~h}$ postoperatively. No significant differences $(\mathrm{P}>0.05)$ between study groups in the occurrence of intraoperative hypotension or bradycardia, patient satisfaction with the type of anesthesia or the hospital length of stay.

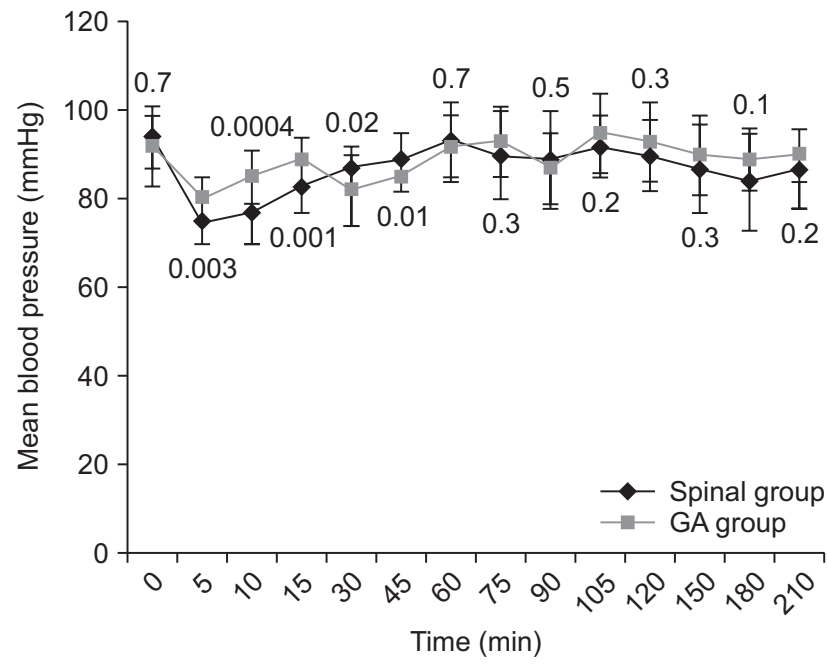

Fig. 1. Comparison of mean arterial blood pressure between the two groups.

However, the mean fentanyl consumption in the GA group was $62.5 \pm 10.6 \mu \mathrm{g}(\mathrm{P}<0.0001)$. Morphine consumption during the first $24 \mathrm{~h}$ postoperatively was significantly greater in the GA than spinal group $(\mathrm{P}<0.0001)$ (Table 2).

In terms of postoperative complications, more patients had vaso-occlusive crises in the GA than spinal group; however, the

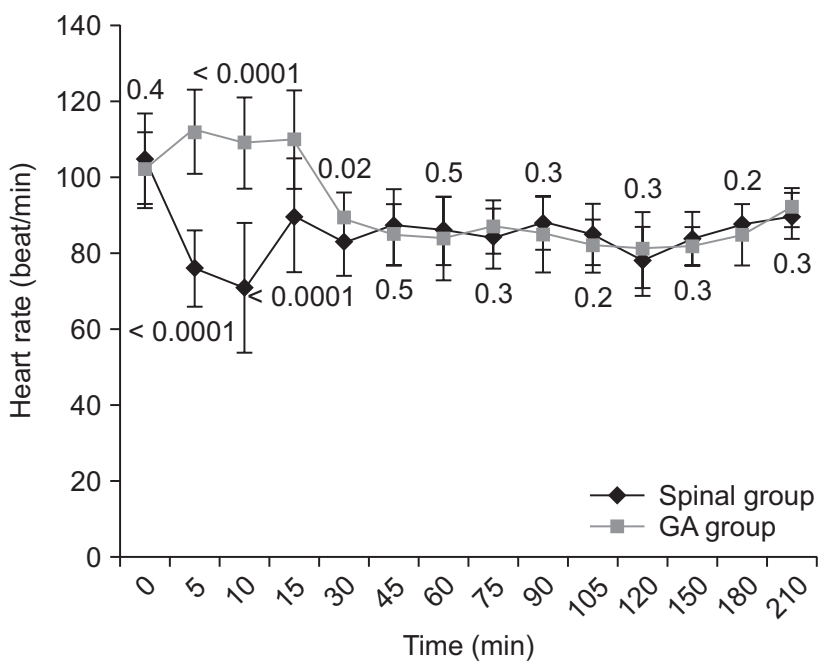

Fig. 2. Comparison of heart rate between the two groups.

difference was not statistically significant $(P=0.4)$. There was one case of acute chest syndrome in the GA group; this patient was admitted to the intensive care unit and managed by oxygen supplementation, good hydration, partial exchange blood transfusion, antibiotic coverage, and aggressive pulmonary therapy, including incentive spirometry. Eventually, the patient improved 
Table 3. Comparison of Neonatal Outcomes between the Two Groups

\begin{tabular}{lccc}
\hline \multicolumn{1}{c}{ Variable } & Spinal group $(\mathrm{n}=20)$ & General anesthesia group $(\mathrm{n}=20)$ & P value \\
\hline Apgar score: & & & \\
At 1 min & $8.13 \pm 1.11$ & $8.21 \pm 0.89$ & 0.006 \\
At 5 min & $9.11 \pm 0.66$ & 7 & 0.009 \\
Admission to neonatal intensive care (n) & 5 & 0 & 0.7 \\
Neonatal mortality during the first week postoperatively (n) & 0 & 1.0 \\
\hline
\end{tabular}

Values are mean \pm SD. There were significant differences $(\mathrm{P}<0.05)$ between study groups in the Apgar score at 1 and 5 min. No significant differences $(\mathrm{P}>0.05)$ between study groups in the neonatal admission to intensive care or neonatal mortality during the first week postoperatively.

and was discharged on the ninth postoperative day. During the first 24 h postoperatively, more women complained of PONV, but the difference between the study groups was statistically insignificant (Table 2). None of the patients in either group had postdural puncture headache. More women were satisfied with spinal anesthesia than GA, but the difference was not statistically significant (Table 2). The mean hospital length of stay was 1 day longer in the GA than spinal group without statistical significance (Table 2).

On the other hand, the present study showed that the Apgar score was significantly better in the spinal than GA group at 1 and $5 \min (\mathrm{P}=0.006$ and $\mathrm{P}=0.009$, respectively). However, admission to the neonatal intensive care unit was not statistically significant between the two study groups $(\mathrm{P}=0.7)$ (Table 3$)$. There was no neonatal mortality until all mothers were discharged from the hospital (Table 3).

\section{Discussion}

In the present study, spinal anesthesia had better maternal and fetal outcomes than GA in parturients with SCA undergoing CS. GA was identified as a risk factor for maternal complications in women with SCA. Camous et al. [3] considered GA to be a risk factor for postnatal sickling (acute chest syndrome, vaso-occlusive crisis, and stroke). However, neuraxial anesthesia was not identified.

Regional (spinal) anesthesia has several potential advantages over GA in parturients with SCA. These beneficial effects of spinal anesthesia may be related to vasodilatation and venous blood pooling. Vasodilatation enhances the blood flow in the anesthetized area. Therefore, it provides optimal control of pain, which is a common complaint in patients with SCA. Indeed, vaso-occlusive crisis during labor reported to be managed using regional analgesia [6].

The estimated intraoperative blood loss volume was significantly lower in the spinal than GA group. This finding is in agreement with that of Lertakyamanee et al. [7], who reported that spinal anesthesia resulted in significantly less blood loss and higher postoperative hematocrit levels than GA for CS. Addi- tionally, Martin et al. [8] found that women who underwent CS under spinal anesthesia had a significantly less estimated blood loss volume and a lower need for blood transfusion than those who underwent GA.

The main disadvantages of spinal anesthesia are the occurrence of hypotension and bradycardia after the block and the risk of postdural puncture headache. No single patient in our spinal group complained of postdural puncture headache. This may be attributed to the use of a small (25-G) pencil-point spinal needle.

In this study, the mean arterial blood pressure and mean heart rate were significantly lower in the spinal group after the block than in the GA group. However, this decrease in these hemodynamic parameters was within the acceptable range without any clinical significance. Therefore, adequate hydration before spinal anesthesia, uterine displacement, and prompt diagnosis and treatment of hypotension with fluid boluses and ephedrine are important measures to minimize the duration and occurrence of hypotension. Interestingly, despite the vasoconstriction caused by ephedrine, which is critical for patients with SCA, Camous et al. [3] found that ephedrine was not a risk factor for postnatal sickling complications in parturients with SCA.

The short period of hypotension that follows spinal anesthesia may alter the maternal and neonatal acid-base values. However, it does not appear to affect the neurobehavioral performances of the newborn [9]. Rasooli et al. [10] recently found no significant correlation between the changes in the maternal mean blood pressure with the neonatal Apgar scores and umbilical artery acid-base status.

Furthermore, Maayan-Metzger et al. [11] reported the prevalence of moderate and severe decreases in the maternal blood pressure, particularly with regional anesthesia. Surprisingly, they reported that neonates tolerated the change in the placental blood perfusion in a rather benign manner. Furthermore, Juhani et al. [12] reported that the hypotension that occurred before the delivery showed no correlation with low Apgar scores or with low $\mathrm{pH}$ in the umbilical artery of the neonates. The Apgar score and umbilical artery $\mathrm{pH}$ provide the best measures of neonatal outcomes after CS under GA and spinal anesthesia. The score 
at 1 min definitely has value for assessing the effects of different drugs given to the mother during CS [13]. We did not measure the umbilical artery $\mathrm{pH}$; however, we noticed that the Apgar scores at 1 and 5 min were significantly higher in neonates in the spinal than GA group. This finding could be explained by the small dose of drugs required to induce spinal anesthesia, which was unlikely to produce systemic effects in the baby. Consequently, less neonatal exposure to the depressant drugs used in GA exists, with little incidence of iatrogenic hypoxia. One of the important advantages of spinal anesthesia is the decrease in the risk of maternal pulmonary aspiration that might occur during GA, leading to hypoxia and precipitation of acute chest syndrome in parturients with SCA. Mancuso et al. [14] found that the neonatal Apgar scores at 1 and 5 min were significantly higher in the spinal group, and the need for neonatal-assisted ventilation was lower in the spinal than GA group. Abdallah et al. [15] recently reported that the Apgar scores at 1 and $5 \mathrm{~min}$ were significantly higher in newborns of parturients who received regional anesthesia than in neonates who were delivered under GA. They correlated their findings to the effect of transient sedation secondary to the GA medications.

In contrast, Kavak et al. [16] revealed that there was no sig- nificant difference in the occurrence of perinatal stress (arterial $\mathrm{pH}$, bicarbonate level, partial pressure of oxygen, and partial pressure of carbon dioxide) in neonates who were born under spinal or GA. They concluded that the type of anesthesia did not influence the short-term outcome of newborns in elective CS.

Finally, as shown by other researchers [12,17], the general advantages of spinal anesthesia over GA for CS include its simplicity, small drug dose, an awake mother, minimal depression of the newborn, and avoidance of the risks of GA, particularly failed intubation and aspiration pneumonitis, which occur at a higher frequency in the maternal population than in nonobstetrical patients.

In conclusion, spinal anesthesia may have potential advantages over GA in parturients with SCA undergoing CS because of less blood loss during surgery, lower intraoperative and postoperative analgesic requirements, and a better Apgar score.

\section{Study limitations}

This pilot study had a small sample size. Further randomized studies with larger sample sizes are needed to confirm the findings of this study.

\section{References}

1. Silva-Pinto AC, de Oliveira Domingues Ladeira S, Brunetta DM, De Santis GC, de Lucena Angulo I, Covas DT. Sickle cell disease and pregnancy: analysis of 34 patients followed at the Regional Blood Center of Ribeirão Preto, Brazil. Rev Bras Hematol Hemoter 2014; 36: 329-33.

2. Koshy M, Burd L. Management of pregnancy in sickle cell syndromes. Hematol Oncol Clin North Am 1991; 5: 585-96.

3. Camous J, N'da A, Etienne-Julan M, Stéphan F. Anesthetic management of pregnant women with sickle cell disease-effect on postnatal sickling complications. Can J Anaesth 2008; 55: 276-83.

4. Brousse V, Makani J, Rees DC. Management of sickle cell disease in the community. BMJ 2014; 348: g1765.

5. Black AS, Newcombe GN, Plummer JL, McLeod DH, Martin DK. Spinal anaesthesia for ambulatory arthroscopic surgery of the knee: a comparison of low-dose prilocaine and fentanyl with bupivacaine and fentanyl. Br J Anaesth 2011; 106: 183-8.

6. Danzer BI, Birnbach DJ, Thys DM. Anesthesia for the parturient with sickle cell disease. J Clin Anesth 1996; 8: 598-602.

7. Lertakyamanee J, Chinachoti T, Tritrakarn T, Muangkasem J, Somboonnanonda A, Kolatat T. Comparison of general and regional anesthesia for cesarean section: success rate, blood loss and satisfaction from a randomized trial. J Med Assoc Thai 1999; 82: 672-80.

8. Martin TC, Bell P, Ogunbiyi O. Comparison of general anaesthesia and spinal anaesthesia for Caesarean section in Antigua and Barbuda. West Indian Med J 2007; 56: 330-3.

9. Corke BC, Datta S, Ostheimer GW, Weiss JB, Alper MH. Spinal anaesthesia for Caesarean section. The influence of hypotension on neonatal outcome. Anaesthesia 1982; 37: 658-62.

10. Rasooli S, Moslemi F. Apgar scores and cord blood gas values on neonates from cesarean with general anesthesia and spinal anesthesia. J Anal Res Clin Med 2014; 2: 11-6.

11. Maayan-Metzger A, Schushan-Eisen I, Todris L, Etchin A, Kuint J. Maternal hypotension during elective cesarean section and short-term neonatal outcome. Am J Obstet Gynecol 2010; 202: 56.e1-5.

12. Juhani TP, Hannele H. Complications during spinal anesthesia for cesarean delivery: a clinical report of one year's experience. Reg Anesth 1993; 18: 128-31

13. Solangi SA, Siddiqui SM, Khaskheli MS, Siddiqui MA. Comparison of the effects of general vs spinal anesthesia on neonatal outcome. Anaesth Pain \& Intensive Care 2012; 16: 18-23.

14. Mancuso A, De Vivo A, Giacobbe A, Priola V, Maggio Savasta L, Guzzo M, et al. General versus spinal anaesthesia for elective caesarean sections: effects on neonatal short-term outcome. A prospective randomised study. J Matern Fetal Neonatal Med 2010; 23: 1114-8. 
15. Abdallah MW, Elzayyat NS, Abdelhaq MM, Gado AM. A comparative study of general anesthesia versus combined spinal-epidural anesthesia on the fetus in Cesarean section. Egyptian Journal of Anaesthesia 2014; 30: 155-60.

16. Kavak ZN, Başgül A, Ceyhan N. Short-term outcome of newborn infants: spinal versus general anesthesia for elective cesarean section. A prospective randomized study. Eur J Obstet Gynecol Reprod Biol 2001; 100: 50-4.

17. Eltzschig HK, Lieberman ES, Camann WR. Regional anesthesia and analgesia for labor and delivery. N Engl J Med 2003; 348: 319-32. 